\title{
Investigation of laser-powder interaction in laser powder bed fusion process in additive manufacturing
}

\author{
Erlei Li, Lin Wang, Ruiping Zou, Aibing Yu, and Zongyan Zhou* \\ ARC Research Hub for Computational Particle Technology, Department of Chemical Engineering, Monash University, Clayton, VIC \\ 3800, Australia
}

\begin{abstract}
Laser powder bed fusion (LPBF) is one of the most promising additive manufacturing (AM) technologies to fabricate metal components using laser beams. To understand the underlying thermal and physical phenomena in LPBF process, discrete element method (DEM) is applied to generate the randomly packed powder, then computational fluid dynamics (CFD) coupled with volume of fluid (VOF) is adopted to simulate the laser-powder interaction. The penetration and multiple reflection of laser rays is traced. The physics of melting and solidification is captured. The temperature profile indicates the laser travel path and the adsorption and transmission of laser rays with the powder. The wetting behaviour of the melt pool driven by the capillary forces leads to the formation of pores at the connection zone. It has been demonstrated that the developed model can capture the laser-powder interaction for further understanding of LPBF process.
\end{abstract}

\section{Introduction}

Laser powder bed fusion (LPBF) is one of the additive manufacturing (AM) technologies, which is fast developing in recent years for industrial metal production in aerospace [1] and biomedical sectors [2]. In LPBF process (particularly the selective laser melting), thin layers of metal powder are distributed by a spreader (blade or roller). Then Laser beam with high energy density is applied to melt the powder, following the process that melted regions solidify and fuse together to form a layer of the built component. The process of powder deposition, melting and solidification is repeated until achieving the final part.

Although the LPBF technology has been widely applied, it still faces challenges in controlling fabricated part quality that is affected by the defect formation (e.g., balling effects, lack of fusion and pores) [3]. These undesired generated defects are closely related to the thermophysical phenomena such as heat transfer in powder bed, melting of powder, droplet spattering and evaporation which are resulted from laser-powder interaction. Understanding the physics and defects formation mechanisms in LPBF is crucial for production control. Research work has been taken in the past years. For example, X-ray imaging is used to capture the laserpowder interaction directly while it is limited by the spatial resolution [4]. Modelling is an alternative method to obtain the detailed information such as the temperature and velocity in melting and solidification, for example, as reviewed in [5, 6]. In our recent work [7], a three-phase model on the basis of CFD coupled with volume of fluid (VOF) was developed to capture the melt pool dimensions and melt pool dynamics. In such a model, important terms are considered such as the mushy zone, surface tension, Marangoni effect and recoil pressure. Laser ray tracing model with multiple reflection is interpolated to represent the laser rays and discrete transfer radiation model (DTRM) is employed to describe the adsorption and transmission between laser rays and powder. Thus, it is able to to capture the melt pool flow dynamics and thermal behaviour in the melting and solidification process. However, the powder layer used is a square-packed array, and also the detailed analysis of laser-powder interaction is lacking in our previous work [7]. Hence, this work aims to address the two issues, in particular, the random powder bed is generated by discrete element method (DEM).

\section{Model description}

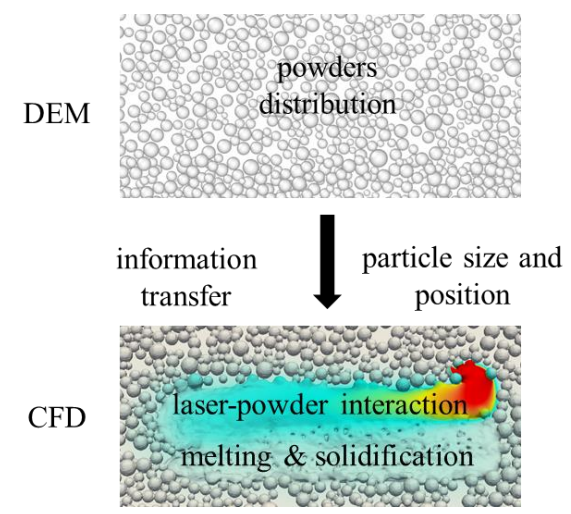

Fig. 1. Framework for the LPBF modelling: DEM is used to generate powder layers (the top), and CFD is applied to capture the melting/solidification of powder (the bottom).

Powder bed deposition and melting and solidification are the two main steps of LPBF process. Therefore, in

* Corresponding author: zongyan.zhou@monash.edu

A video is available at https://doi.org/10.48448/x797-vy17 
this work, the powder bed deposition is produced by DEM which gives the detailed information of individual powder such as the particle size and positions [8]. This detailed particle information is then transferred to CFD model [7] to simulate the melting and solidification process, and the framework is shown in Fig. 1.

The governing equations for the modelling of DEM and CFD and simulation conditions are briefly described below.

\subsection{Governing equations}

The detailed governing equations about powder bed spreading in DEM can be seen in [8]. For CFD model, VOF with mass transfer (melting and solidification mass transfer) is utilized to track the free surface at different phases and its governing equation is expressed as:

$$
\frac{\partial \alpha}{\partial t}+\nabla \cdot(\alpha \mathbf{U})=0
$$

where $\alpha$ is the volume fraction of each phase, $\mathbf{U}$ represents the velocity. Note that the melting mass transfer rate is controlled by the melting coefficient:

$$
\dot{m}_{s l}=\beta_{s l} \rho_{s} \alpha_{s} \frac{\left(T-T_{l}\right)}{T_{l}} \text { for } T>T_{l}
$$

where $\dot{m}_{s l}, \beta_{s l}, \alpha_{s} T$ and $T_{l}$ respectively represent the melting mass transfer rate, melting coefficient, solid phase fraction, temperature and liquidus temperature.

The molten liquid is assumed to be incompressible, and mushy zone (the solid-liquid mixture region formed in the melting and solidification process), surface tension, Marangoni force (resulted from surface tension variation caused by temperature gradient) and recoil pressure (a force generated on the liquid-gas interface when the temperature is higher than the boiling point) are considered in the momentum equation:

$$
\begin{gathered}
\frac{\partial(\rho \mathbf{U})}{\partial t}+\nabla \cdot(\rho \mathbf{U U})=-\nabla p+\rho \mathbf{g}+\nabla \cdot\left(\mu\left(\nabla \mathbf{U}+\nabla \mathbf{U}^{T}\right)\right) \\
+C \frac{\left(1-\alpha_{l}\right)^{2}}{\alpha_{l}{ }^{3}+a} \mathbf{U}+\sigma \kappa \nabla \alpha+\frac{d \sigma}{d T}(\nabla T-\boldsymbol{n}(\boldsymbol{n} \cdot \nabla T))|\nabla \alpha| \\
\quad+0.54 P_{0} \exp \frac{L_{v} M\left(T-T_{v}\right)}{R T T_{v}} \nabla \alpha
\end{gathered}
$$

where $\rho$ is the density, $p$ is the pressure, $\mathbf{g}$ is the gravity acceleration, $\mu$ is the dynamic viscosity, $C$ is a constant related to mushy zone, $\alpha_{l}$ is the liquid volume friction, $\sigma$ is the surface tension, and the last two terms correspond to the Marangoni force and recoil pressure, respectively.

Latent heat of fusion, convective heat transfer, radiative heat transfer and input energy from laser are include in the energy conservation:

$$
\begin{gathered}
\frac{\partial\left(\rho c_{p} T\right)}{\partial t}+\nabla \cdot\left(\rho C_{p} T \mathbf{U}\right)=\nabla \cdot(k \nabla T)-L_{f}\left(\frac{\partial\left(\rho_{l} \alpha_{l}\right)}{\partial t}+\nabla \cdot\right. \\
\left.\left(\rho_{l} \alpha_{l} \mathbf{U}\right)\right)-h_{c}\left(T-T_{r e f}\right)-\sigma_{s b} \varepsilon_{e}\left(T^{4}-T_{r e f}^{4}\right)+S \quad(4),
\end{gathered}
$$

where $C_{p}$ is specific heat capacity, $k$ represents thermal conductivity, $L_{f}$ is the latent heat of fusion, $h_{c}$ is the convective heat transfer coefficient, $\sigma_{s b}$ is the StefanBoltzmann constant, $\varepsilon_{e}$ is the emissivity, and $S$ is the input laser energy considering the multiple reflection. Note that in the laser ray tracing model, a set of collimated rays are traced when they travel through the medium. In DTRM, the change of laser energy density along the travel path is calculated by the radiative transfer equation.

More details about the CFD governing equations including the VOF with mass transfer, continuity and momentum conservations, energy equation and laser energy with multiple reflections can be found in [7].

\subsection{Simulation conditions}

A random distributed powder bed with a powder size distribution in the range of $15-45 \mu \mathrm{m}$ and $d_{50}$ of $30 \mu \mathrm{m}$ is applied in this work. Material of Ti-6Al-4V is adopted to simulate its interaction with the laser rays. The key parameters such as powder properties and model parameters are listed in Table 1. The computational region consists of the substrate, powder bed and gas. The laser scans along the positive $\mathrm{X}$ axis (the red arrow line shown in Fig. 2).

Table 1. Key parameters used in the simulation.

\begin{tabular}{|c|c|}
\hline Parameters & Values \\
\hline Density of solid $\left(\rho_{s}\right)$ & $4420 \mathrm{~kg} \mathrm{~m}^{-3}$ \\
\hline Density of liquid $\left(\rho_{l}\right)$ & $3682 \mathrm{~kg} \mathrm{~m}^{-3}$ \\
\hline $\begin{array}{c}\text { Specific heat capacity of solid } \\
\left(C_{p s}\right)\end{array}$ & $670 \mathrm{~J} \mathrm{~kg}^{-1} \mathrm{~K}^{-1}$ \\
\hline $\begin{array}{c}\text { Specific heat capacity of liquid } \\
\left(C_{p l}\right)\end{array}$ & $831 \mathrm{~J} \mathrm{~kg}^{-1} \mathrm{~K}^{-1}$ \\
\hline $\begin{array}{c}\text { Specific heat capacity of gas } \\
\left(C_{p g}\right)\end{array}$ & $520.32 \mathrm{~J} \mathrm{~kg}^{-1} \mathrm{~K}^{-1}$ \\
\hline $\begin{array}{c}\text { Thermal conductivity of solid } \\
\left(k_{s}\right)\end{array}$ & $10.6 \mathrm{~W} \mathrm{~m} \mathrm{~K}^{-1}$ \\
\hline $\begin{array}{c}\text { Thermal conductivity of liquid } \\
\left(k_{l}\right)\end{array}$ & $33.4 \mathrm{~W} \mathrm{~m}^{-1} \mathrm{~K}^{-1}$ \\
\hline $\begin{array}{c}\text { Thermal conductivity of gas } \\
\left(k_{g}\right)\end{array}$ & $1.772 \times 10^{-2} \mathrm{~W} \mathrm{~m}^{-}$ \\
$1 \mathrm{~K}^{-1}$
\end{tabular}

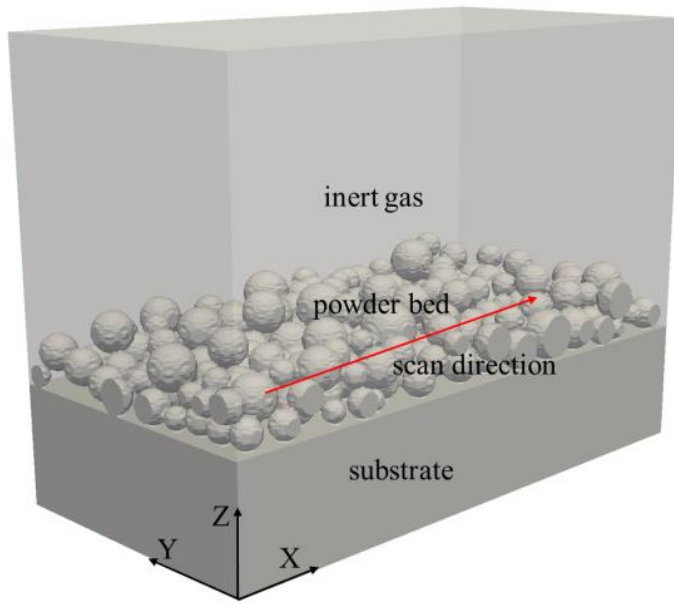

Fig. 2. Computational domain of $500 \mu \mathrm{m} \times 250 \mu \mathrm{m} \times 370 \mu \mathrm{m}$ which consists a $100-\mu \mathrm{m}$ tall substrate, a $70-\mu \mathrm{m}$ tall powder bed and a $200-\mu \mathrm{m}$ tall inert gas region.

To satisfy the CFL (Courant Friedrichs Lewy) condition, time step of $10^{-8} \mathrm{~s}$ is chosen first, and it can be adjusted automatically to make the Courant number lower than 1.0 in the calculation process. The initial temperature field inside the simulation domain is set to be $300 \mathrm{~K}$. The temperature boundary conditions for bottom surface and other faces are fixed temperature of $300 \mathrm{~K}$ and zero gradient, respectively. All faces are set 
as zero for velocity except the left face with a fixed value of $1 \mathrm{~m} / \mathrm{s}$.

\section{Results and discussion}

Capturing the laser-powder interaction and realistic representation of heat deposition on the powder bed are the advantages of the model. This model has been validated by the data of melt pool width and surface roughness from experiments [7]. Note that this work is based on the bed with random distributed powder which is quite different to [7] using the uniform and ordered powder arrangement. Besides, the physics of laserpowder interaction and partial melting of powder has not been fully revealed by our previous work [7].

To observe the laser-powder interaction directly, the travel path of laser rays is illustrated in Fig. 3. At the beginning, four collimated laser beams shot to the system and they pass through the gas media (see Fig. 3(a)) and hit the solid powder surface (see Fig. 3(b) where the solid surface is changed to concave due to the mass transfer to liquid, which indicates that only the region under the initial laser-powder interaction is melted). Based on the random distributed powder and the spherical morphology of particles, multiple reflection is generated as shown in Fig. 3(b) where laser beams scatter to surrounding powder and gas domain. This physics can increase the laser energy adsorption [8]. The laser rays penetrate to the powder deeply, even to the substrate presented in Fig. 3(a). It can lead to substrate melting which will be discussed in the following section.

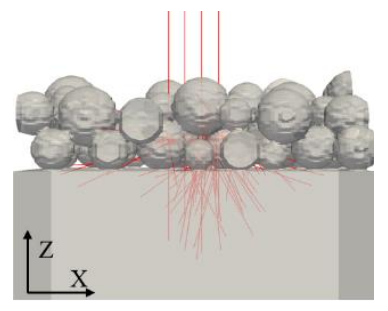

(a)

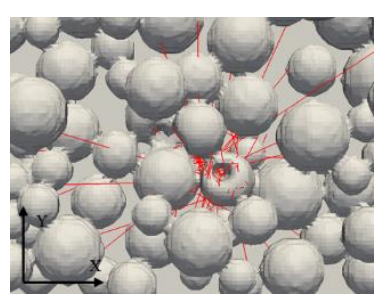

(b)
Fig. 3. The travel path of laser rays: (a) penetration of rays in the $\mathrm{X}-\mathrm{Z}$ plane; and (b) top view of multiple reflection in the $\mathrm{X}$ $\mathrm{Y}$ plane. The laser rays are indicated by the red lines.

The powder interaction with the laser rays (the laser scans from the bottom to the top) is presented in Fig. 4. At the beginning, the laser rays touch the powder, and the temperature increases rapidly to the melting point of $1984 \mathrm{~K}(t=200 \mu \mathrm{s})$. The adsorbed heat energy reallocates in the powder bed from the laser spot centre to surrounding media by the heat conduction. After 25 $\mu \mathrm{s}$, the powder which interacts with laser rays first is melted partially. This makes the substrate exposed in the laser beam and the melting behaviour can penetrate deeper. With the laser moving forward, the first powder interacted with laser is completely melted and the powder on the left of this one is partially melted because of the limited range of laser beam energy $(t=250 \mu \mathrm{s})$. The particles at the edge of the laser diameter are heated while the temperature of these particles is lower than the melting point, hence keeping their original spherical shape which can be observed at $t=250,275$ and $300 \mu \mathrm{s}$ in Fig. 4. Due to the different layer thickness along the laser scanning direction, the melted part in the substrate is uneven and three disconnected concave regions are generated at $t=300 \mu \mathrm{s}$. This phenomenon indicates that the laser ray tracing method used in the model can lead to non-uniform melting and this method is more realistic to represent the laser beams than surface or volume heat source.

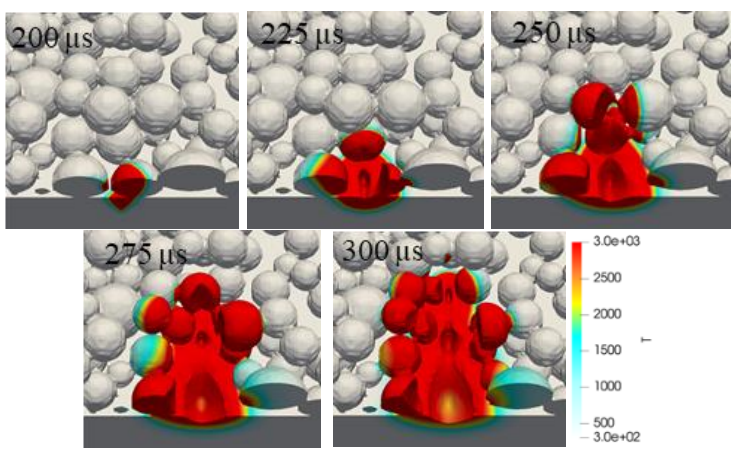

Fig. 4. The process of laser-powder interaction. The laser power is $150 \mathrm{~W}$ with a spot size of $50 \mu \mathrm{m}$ and a scanning speed of $1000 \mathrm{~mm} / \mathrm{s}$.

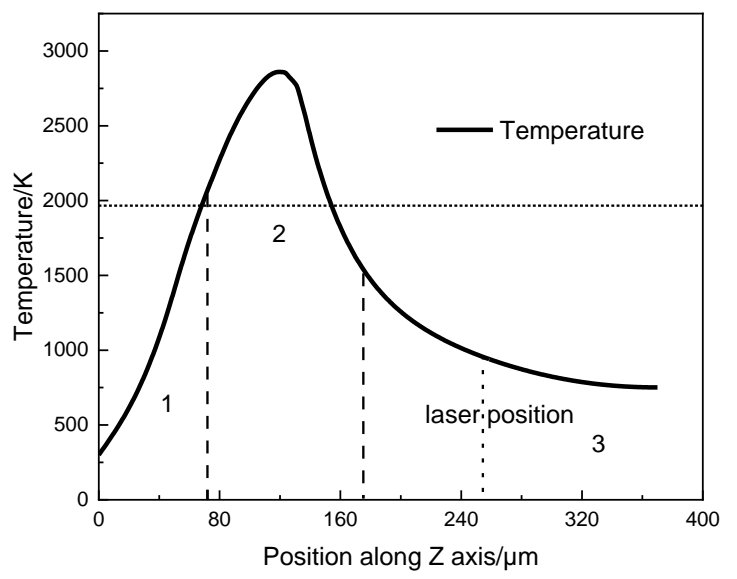

Fig. 5. Temperature profile in the $\mathrm{Z}$ axis (at $\mathrm{X}=440 \mu \mathrm{m}, \mathrm{Y}=$ $125 \mu \mathrm{m})$. The numbers of 1,2 , and 3 correspond to the substrate, powder bed and gas region, respectively (region between dashed lines). The laser's position is indicated by the vertical dotted line. The melting point indicted by the horizontal dotted line is $1948 \mathrm{~K}$. The process parameters are the same as shown in Fig. 4's caption.

To better observe the laser-powder interaction and heat transfer, the temperature distribution along the $\mathrm{Z}$ axis in the simulation domain is collected and shown in Fig. 5. Partial laser energy is absorbed by the gas region, and the left laser rays can transmit through gas and reach to the powder bed which adsorbs most of the heat density, thus presenting the highest temperature ranging from $1500 \mathrm{~K}$ to $2900 \mathrm{~K}$. Note that the temperature in the gas region decreases along the positive direction of $\mathrm{Z}$ axis. Due to the heat conduction and laser penetration, the temperature in some area of the substrate exceeds the melting point which is consistent with the melting results in Fig. 4. The bottom face is set as $300 \mathrm{~K}$ initially as the boundary condition which may affect the temperature distribution in the substrate. Further study 
is needed to investigate the effect of thermal boundary conditions.

The powder melting and melt pool solidification process is illustrated in Fig. 6. The region interacted with the laser rays is melted which keeps the spherical shape $(t=200 \mu \mathrm{s})$ in the beginning. The melted powder presents the fluid dynamics and collapses to the melt pool which can be well observed at $t=250$ and $300 \mu$ s. The molten liquid can wet neighboring powder driven by the capillary forces such as the surface tension and Marangoni effect. This phenomenon is presented at $t=$ 425 and $500 \mu \mathrm{s}$ where the melt pool connects with the two adjacent powder on the left and one large powder on the right. The molten liquid spreads and bounds together with surrounding powder, which results in the pores $(t=1000 \mu \mathrm{s})$ which are generated at the connection zone (edge of the melt pool) after fully solidifying. Theses pores could be eliminated by multitrack scanning.

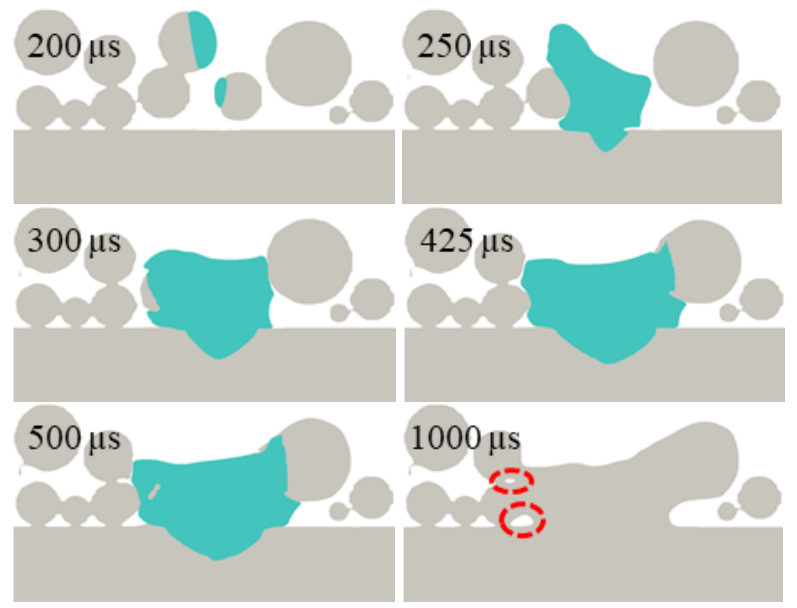

Fig. 6. Snapshots of melting and solidification process in the Y-Z plane $(X=220 \mu \mathrm{m})$. The process parameters are the same as shown in Fig. 4's caption, and the laser turns off at $440 \mu$ s. The melt pool and formed porosities are indicated by the shallow blue contour and red dashed circle, respectively.

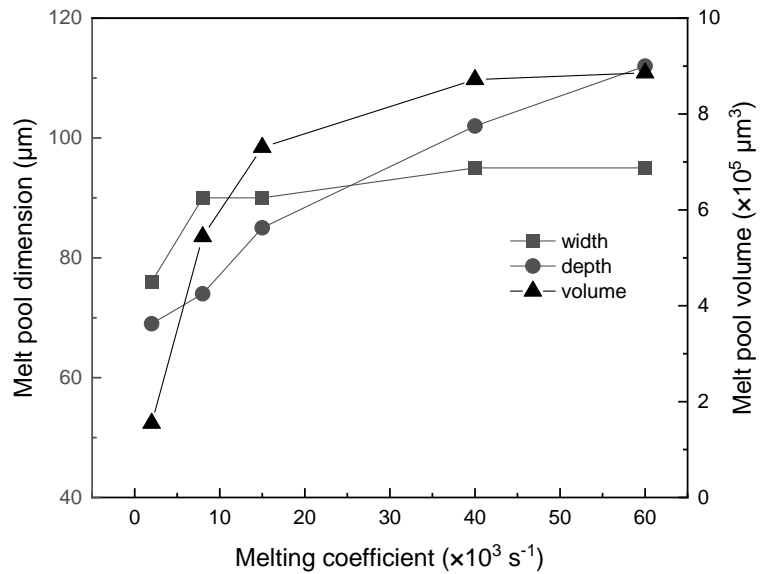

Fig. 7. Melt pool dimension and volume at different melting coefficients at $425 \mu \mathrm{s}$. The process parameters are the same as shown in Fig. 4's caption.

Note that the phase change from solid powder to molten liquid occurs in LPBF process and the mass transfer rate is controlled by the melting coefficient [7]. Fig. 7 gives the melt pool dimension and melt pool volume with various values of melting coefficient ranging from $2 \times 10^{3} \mathrm{~s}^{-1}$ to $6 \times 10^{4} \mathrm{~s}^{-1}$. Results show that the melt pool dimension of width and depth, and the melt pool volume increase with the increase of melting coefficient. It indicates that the increase of melting coefficient enlarges the melting mass transfer rate. For example, the volume grows from $1.55 \times 10^{5} \mu \mathrm{m}^{3}$ to $8.86 \times 10^{5} \mu \mathrm{m}^{3}$ as the melting coefficient increases from $2 \times 10^{3} \mathrm{~s}^{-1}$ to $6 \times 10^{4} \mathrm{~s}^{-1}$.

\section{Conclusions}

The laser-powder interaction in LPBF process is studied in this work where DEM provides particle positions and CFD is used to simulate the melting and solidification process. The travel path of laser rays in the system is traced, and the penetration and multiple reflection of laser rays is illustrated. The thermal and physical phenomena such as powder full and partial melting are well captured. Non-uniform melting is observed due to the spatial layer thickness variation on the random distributed powder bed. The temperature distribution in the simulation domain indicates the laser travel path and the interaction between laser rays and the media including the gas, powder and substrate. The wetting dynamics of melt pool and pores formation mechanism are revealed. The increase of melting coefficient enlarges the melting mass transfer rate. This model can capture the main features in LPBF process, but the model still needs further improvement to enable to reveal metal evaporation and laser-vapor interaction.

The authors are grateful for the financial support from Australian Research Council, and the National Computational Infrastructure for computation.

\section{References}

[1] Z.L. Lu, J.W. Cao, H. Jing, T. Liu, F. Lu, D.X. Wang, D.C. Li, Virtual Phys. Prototyp. 8, 87-95 (2013)

[2] J.E. Biemond, G. Hannink, N. Verdonschot, P. Buma, J Mater. Sci. Mater. Med. 24, 745-753 (2013)

[3] B. Zhang, Y. Li, Q. Bai, Chin. J. Mech. Eng. 30, 515527 (2017)

[4] C.L.A. Leung, S. Marussi, R.C. Atwood, M. Towrie, P.J. Withers, P.D. Lee, Nat. Commun. 9, 1-9 (2018)

[5] P.S. Cook, A.B. Murphy, Addit. Manuf. 31, 100909 (2020)

[6] H.L. Wei, T. Mukherjee, W. Zhang, J.S. Zuback, G.L. Knapp, A. De, T. DebRoy, Prog. Mater. Sci. 116, 100703 (2021)

[7] E.L. Li, L. Wang, A.B. Yu, Z.Y. Zhou, Powder Technol. 381, 298-312 (2021)

[8] L. Wang, E.L. Li, H. Shen, R.P. Zou, A.B. Yu, Z.Y. Zhou, Powder Technol. 363, 602-610 (2020) 